\title{
Effects of an Internationalization at Home (IAH) Programme on Cultural Awareness among Medical and Nursing Students in Hong Kong and Indonesia During the COVID-19 Pandemic: A Mixed-Methods Study
}

Patrick Pui Kin KOR ( $\nabla$ patrick.kor@polyu.edu.hk)

Hong Kong Polytechnic University

Clare YU

University College London

Ida ayu TRIASTUTI

Duta Wacana Christian University

Mitra Andini SIGILIPOE

Duta Wacana Christian University

Haryo Dimasto KRISTIYANTO

Duta Wacana Christian University

Johana Puspasari Dwi PRATIWI

Duta Wacana Christian University

Teguh Kristian PERDAMAIAN

Duta Wacana Christian University

Mengli Lisa LI

Hong Kong Polytechnic University

Phyllis PANG

Hong Kong Polytechnic University

Maria Meiwati WIDAGDO

Duta Wacana Christian University

Research Article

Keywords: Internationalization at home, medical education, cultural sensitivity, cultural awareness.

Posted Date: January 12th, 2022

DOI: https://doi.org/10.21203/rs.3.rs-1187682/v1 
License: (c) (i) This work is licensed under a Creative Commons Attribution 4.0 International License. Read Full License 


\section{Abstract}

Background: The COVID-19 pandemic has severely impacted the learning experience of students by limiting their opportunities for face-to-face intercultural exchanges. Given the importance of cultural competence in medical education, there is a need to develop a programme that promotes cultural awareness, but that offers more flexibility in terms of outbound mobility. This study aims to evaluate the effectiveness of an internationalization at home programme and to explore the learning experiences of medical and nursing students from Hong Kong and Indonesia.

Methods: Students were recruited from two universities in Hong Kong and Indonesia. They attended an online internationalization at home programme designed by members of the research team from both countries. A mixed-methods study was conducted. A pre-test post-test design was used to evaluate the effects of the programme on cultural awareness, and four focus group interviews were conducted to explore the students' experiences in the programme. Quantitative and qualitative data were analysed by Ttest and thematic analysis. Data were integrated via triangulation.

Results: One hundred and forty-eight students from Hong Kong and Indonesia participated in the study. After the programme, there was a significant improvement in cultural awareness. Three themes were identified: (1) learning process: enjoyable, but a desire remains for face-to-face cross-cultural communication; (2) learning outcomes: gained cultural awareness, developed cultural sensitivity, had an opportunity to practice language and learn about new learning styles; (3) factors influencing learning outcomes: facilitators (micro-movie and active communication) and barriers (language barrier, inappropriate time arrangement, insufficient prior briefing).

Conclusion: This programme achieved the learning outcomes by successfully enhancing the cultural awareness of students during a time of pandemic when outbound student exchanges were not possible. Further adaptations of the programme are required to further enhance learning outcomes.

\section{Introduction}

Due to globalization, the population of every country has become more culturally diverse. Healthcare professionals often need to interact with individuals whose health beliefs and life experiences are very different from their own. To meet the healthcare needs of people from different cultures, cultural competency is increasingly being regarded as an essential skill in medical training [1]. Previous studies have suggested that cultural competency may improve communication between healthcare professionals and patients, increase the satisfaction and treatment adherence of patients, and improve clinical outcomes [2].

Cultural competency can be defined as the ability to provide care to patients of diverse values, beliefs, and behaviors, including tailoring healthcare delivery to meet the demands of patients [3]. Cultural competency can be broadly conceptualized as comprised of three concepts: 1.) Awareness of one's own culturally related biases and values, 2.) Knowledge about the cultural values of diverse populations, and 
3.) Skills that can be applied when working with a culturally diverse patient group [3]. Previous studies have suggested that students can acquire cultural competency through education [1]. Students equipped with cultural competency might well be more readily prepared to accept challenges related to cultural diversity in clinical settings.

Nowadays, cultural competency training is often a component of university curricula [1]. Such training often involves providing direct multicultural experiences, such as through an overseas exchange or overseas placement. Through such face-to-face cultural exchange programmes, students can practice in settings and with cultures other than their own. However, this approach is often heavily criticized by people in the field because of its inflexibility with regard to student mobility (students must travel overseas in order to be exposed to this education). Not only is this approach inherently inequitable for the financially disadvantaged, but this type of programme also fails completely when border restrictions are in place, such as during the Covid-19 pandemic. Hence, there is a need for an internationalization at home programme $(\mathrm{IAH})[4,5]$.

Internationalization at home is a newer concept that aims to provide students with international skills and experiences without the need to be involved in a travel exchange. The concept of IAH is 'the purposeful integration of international and intercultural dimensions into the formal and informal curriculum for all students within a domestic learning environment' [6]. IAH has been implemented by several institutions of higher education. In 2017, 31 nursing students from Australia, Hong Kong, and Sweden participated in a series of IAH programmes. The study showed that the students who participated in the IAH activities became aware of their own professional and personal ethical ideals, and improved their relational skills. IAH activities have also been helpful in empowering students to take the lead and encourage each other to value diversity [7]. On the other hand, a scoping review of the internationalization of medical education in 2020 revealed that although there has been a drastic increase in research in this area in the last 25 years, the number of published peer-reviewed scientific articles on this subject has been limited. In this review, significant findings were limited because only a few schools have engaged in IAH, and comparative data from related professions are lacking, with some schools and professions not focusing on standardized goals [8]. Furthermore, most existing internationalization programmes for nursing/medical students have focused on clinical students, not preclinical students [9].

Considering the on-going pandemic and limited opportunities for physical mobility, IAH has a stronger role than ever to play in empowering cultural competencies. There is an imminent need for effective IAH programmes that allow students to develop intercultural competencies through a virtual platform [10]. The authors conducted a programme that delivered IAH to students in the health professions with robust technology assisting in the preparation, class sessions, and evaluation of the programme. The programme was conducted for students in the medical and nursing professions, with the main objective of promoting cultural awareness. The objectives of this study were to: 1.) Evaluate the effectiveness of an IAH programme in raising the cultural awareness of the students, and 2.) Explore the students' experiences with the IAH programme and to determine facilitators and barriers to the use of the programme. 


\section{Methods}

\section{Design}

This study adopted a concurrent mixed-methods design, which involved the conducting of surveys and interviews. This approach is particularly advantageous when evaluating a relatively new programme such as the IAH, [11], as it made use of multiple sources of data to provide a broader and deeper understanding of the programme. For the quantitative arm, we used a pre-/post-test design to investigate the students' cultural awareness after the programme, whereas the focus group interviews were conducted to explore the experiences of students in the programme. The ethics committee of The Hong Kong Polytechnic University (HSEARS20210604001) approved this study.

\section{Study Participants And Setting}

The participants were nursing students from the School of Nursing, The Hong Kong Polytechnic University and medical students from the Duta Wacana Christian University in Indonesia. Students were recruited from a subject called 'Healthy Lifestyle Challenges for Developing Communities'. This course is an elective at both universities. All of the activities were conducted via the online platform ZOOM.

\section{lah Programme}

To align with the subject objectives in promoting a healthy lifestyle, the subject team drew up the following expected outcomes. After the IAH programme, students will be able to:

1. examine how local and global contexts intersect in managing health-related problems, which include unhealthy eating habits, a lack of physical exercise, and addictive behaviours (e.g., smoking);

2. apply evidence, concepts, and theories to problem cases related to leading an unhealthy lifestyle in different cultural contexts;

3. develop a cross-cultural understanding of the social and cultural determinants of a healthy lifestyle;

4. broaden their international and cross-cultural awareness and intercultural sensitivity;

5. enrich their preparedness in the areas of social responsibility and a global outlook.

Five sessions of an online IAH programme (4 hours each) were provided for the PolyU students and the Indonesian students from Duta Wacana Christian University. To align with the above outcomes, we designed the following teaching and learning activities:

1. To understand how local and global contexts intersect in managing health-related problems, which include unhealthy eating habits, a lack of physical exercise, and addictive behaviours; and to enrich the students' preparedness in the areas of social responsibility and a global outlook 
- An enquiry-based learning (EBL) approach was adopted. A micro-movie was developed to simulate the life of an Indonesian domestic helper in Hong Kong who looks after a Chinese older person. The video portrayed an Indonesian lady living in Hong Kong who helps to take care of an older person. Both the Indonesian lady and the older person had various unhealthy lifestyle habits. For example, the Indonesian lady always eats fast food and junk food, and the older person does not engage in sufficient physical activity. Also, the Indonesian lady faced various difficulties when providing care for the older person with regard to the appropriate diet and types and amount of physical activity because of her lack of knowledge of the needs of older people and cultural differences. In this workshop, students from different cultural backgrounds were asked to form small groups. Together, they searched for and used various resources to address the above problems. The students had to determine what they needed to learn, identify appropriate learning resources, report their learning outcomes, and assess their progress in learning. Through this learning process, the students were expected to understand more about how the same health problem is managed in two different regions among people of different cultural backgrounds, Chinese and Indonesian. During the discussion, the teacher also guided the students to further understand how local and global contexts intersect in managing health-related problems.

\section{To apply evidence, concepts, and theories to problem cases related to leading an unhealthy lifestyle in different cultural contexts; and to develop into a socially responsible global citizen}

- Before the IAH programme, the students had already learnt about health assessment skills, health promotion strategies, global citizenship, and intercultural competence from the lectures and in-class activities. In the programme, the students worked together to develop a health promotion plan for the cases in the mico-movies. The students did not only apply the skills that they had learnt from the lectures, but also needed to learn and discuss with the foreign students about cultural contexts when developing a health promotion plan for the Indonesian and Chinese people. The students had to make a group presentation during the workshop and the different student groups had to critically appraise the health promotion plan developed by the respective peer groups.

3. To develop a cross-cultural understanding of the social and cultural determinants of a healthy lifestyle and to broaden the students' cross-cultural awareness and intercultural sensitivity

- This programme started with an ice-breaking activity by students from the two universities, which involved introducing different traditional foods and lifestyles in Hong Kong and Indonesia, followed by a discussion about their implications for a healthy lifestyle. An elibrary was also set up to keep a record of various open-source teaching materials (e.g., website, videos, news) about health problems relating to unhealthy eating, a lack of physical exercise, and additive behaviours in Indonesia and in Hong Kong. The students were asked to read those pre-workshop materials in the e-library to enhance their social and cultural understanding of a healthy lifestyle.

\section{Data Collection}




\section{Quantitative survey method}

Informed consentwas received from all of the participants before the commencement of data collection. All of the students who were enrolled in the subject 'Healthy Lifestyle Challenges for Developing Communities' were invited to complete an online survey before (T0) and after ( $\mathrm{T} 1$ ) the programme. This survey includes the modified cultural awareness scale [12]. The modified CAS was used to measure the cultural awareness (minimal level of cultural competence) of students in higher education. The scale contains a total of 35 items. The responses are based on a 7-point scale (strongly disagree to strongly agree, with a midpoint choice of no opinion) and one additional response 'does not apply'. This scale generates four subscale scores: 'General educational experience (ranging from 15-105)', 'cognitive awareness (ranging from 7-49)', 'clinical issues (ranging from 5-35)', and 'behaviours/comfort with interactions (ranging from 8-56)', and a total score ranging from 35-245. A higher score indicates greater cultural awareness. The scale was demonstrated to have satisfactory validity and reliability among tertiary students from Sweden and Hong Kong in healthcare fields. [12]. Demographic data, including age and gender, were also collected at baseline (TO). The data were analysed using IBM's SPSS (version 25). A paired sample T-test was conducted, and a two sided significance test $(p<.05)$ was applied.

\section{Qualitative Interview Method}

An online consent form was received from all of the participants before the interviews were conducted. Purposive sampling was used to select participants with a range of characteristics, such as study major and year of study that might be related to their learning experience. Four semi-structured focus group interviews were conducted to understand the participants' perceptions of and experiences with the online IAH programme. The Hong Kong and Indonesian students were interviewed separately in Cantonese/Indonesian by three instructors of the course (one Hong Kong and two Indonesian) who had prior knowledge of the internationalization at home pedagogy. In the interview, a guiding question was first employed to open up new testimonies: 'What was your learning experience in taking the online IAH programme?' The participants were then asked about how the experience impacted their study, the elements that they liked/disliked, and the skills, knowledge, and any other benefits that they had gained.

All interviews were audio-recorded on ZOOM and were transcribed and translated into English. A thematic analysis of these transcripts was then performed by two independent researchers (PK and CY) through a collaborative coding process. They developed an initial coding framework by reading a sample of the transcript and discussing it. The two researchers then independently coded all of the remaining transcripts. During this process, the coding framework was revised, with new themes developed or existing themes revised. Disagreements over codes were resolved through discussion between PK and CY.

\section{Data integration}


Quantitative and qualitative data were analysed as described above. Themes generated from the qualitative interviews were considered data that supplemented the findings of the survey. During the integrative analytic process, we compared the qualitative and quantitative findings by tabulating the themes and survey findings (a process of joint displays). The areas of divergence and convergence were discussed iteratively by $\mathrm{CY}$ and PK. These synthesized concepts were then used to understand the learning experience of students in the IAH programme.

\section{Results}

\section{Participants}

A total of 150 students enrolled in the programme. Of these, 148 (98.6\%) completed four sessions, and $\mathrm{n}=148$ completed the pre-test post-test survey. The demographic characteristics of our participants are summarized in Table 1. Characteristics of the participants. Of the students, $67.6 \%(n=100)$ were from Indonesia and $33.1 \%(n=48)$ from Hong Kong. The majority were females $(62.8 \%)$, and their mean age was 21 years ( $S D=1.39$ years). Most $(n=99,83.9 \%)$ were undergraduate students from the discipline of health sciences (i.e., Nursing, Medicine) 
Table 1

Characteristics of the participants $(\mathrm{N}=148)$

Age $(M, S D)$

(21.44,

1.39)

$\mathrm{N}$

(\%)

Gender

Female

$93 \quad 62.8$

Male

$55 \quad 37.2$

Country

Hong Kong

$48 \quad 32.4$

Indonesia

$100 \quad 67.6$

Have you ever visited Indonesia before? *

Yes

$3 \quad 6.3$

No

$45 \quad 93.8$

Have you ever visited Hong Kong before **

Yes

$3 \quad 3.0$

No

$97 \quad 97.0$

Study Major

Applied Science

$\begin{array}{ll}1 & 0.7\end{array}$

Computer Science

$\begin{array}{ll}1 & 0.7\end{array}$

Engineering

$\begin{array}{ll}1 & 0.7\end{array}$

Fashion and Textiles

$\begin{array}{ll}1 & 0.7\end{array}$

Health Sciences (Nursing, Medicine)

$129 \quad 87.1$

Others

$15 \quad 10.1$

Year of study

Year 1

$22 \quad 14.9$

Year 2

$19 \quad 12.2$

Year 3

Year 4

7

4.7

Year 5 or above

$98 \quad 66.2$

$2 \quad 1.4$


Do you have any experience of joining cultural exchange or cultural awareness workshops/training programmes?

Yes

No

*This question was only answered by students from Hong Kong.

** This question was only answered by students from Indonesia.

The majority of our participants (over 93\%) had never visited the partner country/region (Indonesia/Hong Kong). Only $5.4 \%(n=8)$ had attended a cultural exchange programme before, suggesting that our participants from Hong Kong and Indonesia had limited exposure to each other's culture.

\section{Cultural awareness}

The result of the programme's impact on raising cultural awareness is summarized in Table 2 Results of a paired T-test comparing cultural awareness scores before and after the workshop. Our result indicates that after the programme, there was a significant increase in all of the subscale scores on cultural awareness [General education \& research experience $(t(60)=-3.23, p=.00)$, behaviours/comfort with interactions $(t(55)=-2.72, p=.01)$, cognitive awareness $t(65)=-2.22, p=.03)$, clinical issues $t(67)=-3.01$, $\mathrm{p}=.00)]$ and in the total score $(\mathrm{t}(51)=-3.23, \mathrm{p}=.00)$.

Table 2

Results of a paired T-test comparing cultural awareness scores before and after the workshop

\begin{tabular}{|c|c|c|c|c|c|c|c|}
\hline \multirow{3}{*}{$\begin{array}{l}\text { General education \& research } \\
\text { experience }\end{array}$} & \multicolumn{2}{|c|}{ Pre-test score } & \multicolumn{2}{|c|}{ Post-test score } & \multirow[t]{2}{*}{$d f$} & \multirow{2}{*}{$\begin{array}{l}t \\
\text { value }\end{array}$} & \multirow{2}{*}{$\underset{\text { value }}{p}$} \\
\hline & $M$ & $(S D)$ & $M$ & $(S D)$ & & & \\
\hline & 74.10 & 11.07 & 80.39 & 13.85 & 60.00 & -3.23 & 0.00 \\
\hline $\begin{array}{l}\text { Behaviours/comfort with } \\
\text { interactions }\end{array}$ & 31.70 & 7.58 & 35.77 & 9.70 & 55.00 & -2.72 & 0.01 \\
\hline Cognitive Awareness & 35.00 & 5.02 & 36.89 & 5.90 & 65.00 & -2.22 & 0.03 \\
\hline Clinical issues & 25.96 & 4.31 & 28.12 & 4.78 & 67.00 & -3.01 & 0.00 \\
\hline $\begin{array}{l}\text { Cultural awareness score total } \\
\text { score }\end{array}$ & 164.67 & 19.03 & 179.63 & 29.36 & 51.00 & -3.23 & 0.00 \\
\hline \multicolumn{8}{|c|}{$\begin{array}{l}\text { Remarks: The cultural awareness scale (CAS) generated four subscale scores, namely, general } \\
\text { educational experience (subscale score ranging from } 15-105) \text {, behaviours/comfort with interactions } \\
\text { (subscale score ranging from } 8-56 \text { ), cognitive awareness (subscale score ranging from 7-49), and } \\
\text { clinical issues (subscale score ranging from 5-35). It also generated a total score ranging from 35- } \\
245, \text { with a higher score indicating higher cultural awareness. }\end{array}$} \\
\hline
\end{tabular}


A total of 16 students were interviewed in four semi-structured focus group interviews (two each for the Hong Kong and Indonesian students). We identified three major themes in our analysis of the data and present these in each subsection below. The first theme 'learning process' describes students' perceptions and feelings towards the programme. The second theme, 'learning outcomes' describes what outcomes (culture-related and non-culture related) students believed they had achieved from the programme. The third theme, 'factors influencing learning outcomes' describes factors that facilitate and hinder the learning process and learning outcomes. Together, these themes provide context to the general learning experience of the programme. Throughout the analysis, we aimed to provide a sense of the contents of the data by using quotes from anonymized participants, whose country of origin was indicated using the prefix of IN (students from Indonesia) or HK (Hong Kong).

\section{Learning experience}

\section{IAH as an enjoyable experience}

Both the students from Indonesia and Hong Kong described the IAH experience as 'fun', 'interesting', and 'exciting'. These descriptions often came with coding related to a 'novel experience'. Specifically, many students said that this programme enabled them to gain new knowledge of another culture and meet students from another country, and that is what excited them the most.

'It is interesting to discuss culture with people from other countries. In my opinion, it is very interesting. It helps us to widen our horizons and allows us to understand that everyone is different.' (HK-GP1)

'I am excited. I think cultural exchange is an interesting thing. I grasped every opportunity for discussion so that I could get to know them (Hong Kong students) better. I mean ... I was given these opportunities. So, why would I not these opportunities properly? I feel happy and excited because I can meet people from a different background.' (IN-GP 1)

'I am really excited because it is the first time that I have gotten a chance to collaborate with foreigners the Hong Kong students. That teaches me how to work with new people.' (IN-GP 2)

\section{Viewing IAH as a novel experience, but still valuing the essence of face-to-face interactions}

Although students generally appreciated the novelty of the IAH experience and the convenience of not having to travel abroad, in the interviews the students constantly compared the online cultural exchange experience with face-to-face cultural exchange activities. Most indicated that they would still choose faceto-face interactions over an online cultural experience if offered both options.

'I still prefer to know more about the culture by visiting there (Indonesia) locally. That was my goal. I mean ... if I were given the chance to taste the local food there, it would be better for me to learn about their culture.'(HK-GP 2) 
'This programme allows us to learn about the health conditions of other countries even if we don't have to go on a trip. We can still widen our horizons.' (IN-GP 2)

Interestingly, one student described the important role of IAH during the COVID-19 pandemic, when all face-to-face cultural exchange activities were suspended. 'Because of the pandemic, there is no chance to go abroad, so this programme does give us a chance to communicate with students from other countries. It is better than nothing.' (IN-GP 2)

\section{Learning Outcomes}

This theme features learning outcomes that the students believed they had achieved from the programme.

\section{Awareness of cultural diversity (new knowledge, noticing differences, social responsibility)}

Many students said that they gained awareness of cultural diversity after attending the programme. They developed such awareness through gaining knowledge about the health habits and health policies of another country. Such awareness often came as a surprise to the students. One student from Indonesia talked about her awareness of cultural diversity in alcohol addiction. 'My group was responsible for the topic addiction. So we discussed the cultural reasons behind alcohol abuse. Like related laws and regulations. I am surprised that alcohol is available everywhere in Hong Kong and that it is even available in vending machines. The drinking habit there is deeply embedded. For example, alcohol is often served in their company events for the purpose of social bonding, or in family events, so I can imagine that it is harder to manage in Hong Kong.' An Indonesian student also mentioned that she realized there were more informational resources available in Hong Kong. 'They (HK-GP2) have a variety of resources - different educational materials and databases. Here in Indonesia, I think we only have materials from the Health Ministry. When the Health Ministry says something, we always follow along. But they have more than that, and this information is helpful to our project.' (IN-GP1)

Interestingly, from the interviews with students, we realized the new knowledge was not simply coming from interactions within the programme (i.e., discussions, presentations). The students gained new knowledge about another country from social interactions as well, which occurred outside the classroom. For example, a student from Indonesia said that she connected with one of her group mates from Hong Kong via Instagram (a social media platform). 'We read each other's Instagram stories. And I can see that from her story, she was hanging out with her girlfriends and her boyfriend. This is a good exchange. You can learn about their lifestyle, just like health. It really made me become more curious about the Hong Kong culture.' (IN-GP 1)

By raising their cultural awareness, a few students expressed the view that they further developed a sense of international social responsibility. 'I gained more understanding of the health situation in another country, and we learned to view health problems not just from a Hong Konger's perspective. I have learned 
to care about the health issues of other people around the world. Some places require attention from us.' (HK-GP1) A student from Indonesia said, 'It widens our horizons and allows us to understand that everyone is different. At some point, I realized that we have an obligation to help them. We should respect them. You gained insights and thoughts.' (IN-GP2)

\section{Developed cultural sensitivity in clinical practice}

Many students highlighted the view that after attending the programme, they started to realize the importance of cultural sensitivity in clinical practice. Through the activities, especially the group discussions, they learned that cultural sensitivity is always needed when analysing health problems and that cultural adjustments are needed when developing health promotion strategies.

'It widened my horizons. It widened my understanding. It turns out that their practices are different from Indonesians, so for me it's more... In the future, if I have to meet patients who I want to interact with, I am going to understand their cultural background first, how to respect, to understand their culture and also their habits to educate them and give them the appropriate intervention.' (IN-GP2)

'It was fun to share something about our habits and cultures. In the group discussions, we got to know about the lifestyle differences between the two countries. Our plan was to create a change in lifestyle for both Hong Kongers and Indonesians. We discussed what kind of adjustments were needed there, how we should adapt. It was fun!' (INDO-GP1)

They also said that if communication is not culturally sensitive, there is a potential for it to negatively impact the care provided to patients.

'I also feel that it is very important for us to learn about culture, because it will greatly affect the way we communicate with patients and the management of health services.' (INDO-GP2)

\section{Trained up language skills}

Some students mentioned that although they might not speak English well, IAH provided a precious opportunity to practise English.

Many of our local students communicate in Cantonese, but in order for us to interact with the Indonesian students, we cannot use our mother tongue. Our English level may not be good enough. I mean, when you want to express your ideas, you may not be able to articulate well. In the end, we have to use English to explain ourselves. I was able to practise my spoken English. (HK-GP1)

'It is very interesting, speaking English, listening, and learning, and again to talk to foreigners. Yes, in my opinion I gained additional knowledge.' (INDO-GP1)

\section{Adoption of new learning styles}


Some students said that through interactions with students from another country, they learned to model their learning styles and adopted some practices, such as viewing health issues from another perspective, being more proactive in group discussions, and engaging in teamwork.

'I am only a year 1 student. The Indonesian students were able to think of more aspects and perspectives than me. I learned to view things in a different perspective.' (HK-GP1)

'The first thing I noticed is that students from Indonesia really took the initiative to speak up, and they are more proactive than our Hong Kong students, so I started to be more proactive as well in the discussions.' (HK-GP1)

'I have learned the importance of a team in the two-day workshop on internationalization.' (INDO-GP1)

\section{Factors influencing learning outcomes}

From respondents' accounts of what they like and dislike about the workshops, we identified facilitators and barriers affecting learning outcomes in the IAH workshops.

\section{Micro-Movie as a good teaching tool to stimulate discussion}

The micro-movie was constantly mentioned by interviewees as a good teaching tool, as they thought that the movie was 'interesting' (INDO-GP2) and 'stimulating'. They also thought that, in general, it does a good job of depicting cultural differences between Hong Kong and Indonesia.

'The main characters in the movie are the Hong Kong lady and the Indonesian mom. There are a lot of conflicts in their living style. The conflict was especially obvious when both characters lived together.... For example, the mum doesn't eat luncheon meat. It's because there is so much oil, salt, sugar, and so on in it. So, I think the movie captured the difference in eating habits well.' (IN-GP2)

'There was a discussion after the movie. The movie stimulated our discussion. It's enough, to stimulate our discussion about diet, physical activity, addiction. We already know what will be discussed.'(HK-GP1)

Despite this, some students suggested that the micro-movie should place equal focus on the cultures of Hong Kong and Indonesia. An Indonesian student said, 'The characteristics of Indonesia are not outstanding enough in the micro-movie. For example, after I watched the video, I know there are some cultural gaps and cultural differences, but I don't really know what the mom (Indonesian) was not familiar with ... I mean specifically what aspects.... It would be better if the storyline focused more on the Mom.' (IN-GP1)

\section{Active communication as a factor in facilitating cultural exchanges}


Some students mentioned that active communication between group members is a key factor in facilitating discussions about culture. 'For my group, the communication is really good.... All of them are considered active in my opinion. This really helps with the discussion.' (IN)

Although active interactions were being described as a key factor in cultural exchanges, the language barrier was often described as a factor that hindered such interactions. Many students said that there was some awkwardness in their group discussions, as some group members were reluctant to speak English. 'In my group, the problem is more that they don't want to communicate in English ... students from both countries. It is very awkward.' (IN-GP2) 'There are still people at our university who are still not confident in speaking English, even though they are able to do it, but they are shy. (IN-GP1). A few students suggested that further breaking the groups into smaller groups might facilitate more active discussions and interactions. (IN-GP2)

\section{Time management}

Many students mentioned that the time management of the workshop could be improved. They held different opinions about the duration of the activities, with some suggesting that some activities should be longer and some shorter. Most students said that there was limited time (one week) to prepare for the group project presentation, as online collaboration with group members from another country requires extra effort to coordinate.

'Personally, it has something to do with the time, the project time. I think the time is too short to work on a project between students of two countries.' (IN-GP2)

As almost all of the Indonesian students were in their final year, many interviewees from Indonesia expressed the view that the programme schedule didn't fit well into their busy schedule. They thought that the cultural exchange workshop should target junior year students rather than senior year students. 'This workshop should not be placed in the senior year, as it clashes with our thesis and community services.... If it is possible, it should be placed in the second or third year.' (INDO-GP1)

\section{Briefing prior to the workshop}

Some students said they were not well briefed prior to the workshop, so that what they experienced in the workshop was different from what they had expected. Some students expressed disappointment. They said that one improvement would be to give the students a short briefing / workshop briefing note prior to the workshop.

'I thought it was a student exchange programme. But that was different from my initial expectation. It turned out to be a programme for learning each other's culture.' (IN-GP1)

'The overview for this event was a bit sudden (referring to the overview note that the team sent to students prior to the workshop). We didn't know what this event was about.... The downside would be the short notice beforehand.' (IN-GP1) 


\section{Discussions}

Through an analysis of quantitative data and qualitative data, it was found in this mixed-methods study that the IAH programme is effective at improving cultural awareness. Several themes were identified: (1) learning process: enjoyable, but a desire remains for face-to-face cross-cultural communication; (2) learning outcomes: gained cultural awareness, developed cultural sensitivity, had an opportunity to practise language and learn about new learning styles; (3) factors influencing learning outcomes: facilitators (micro-movie and active communication) and barriers (language barrier, inappropriate time arrangement, insufficient prior briefing).

Similar to our study, Leung et al. [7] found that a five-webinar international programme also led to improvements in the overall cultural awareness of postgraduate students, as well as in two dimensions (general educational \& research experience and cognitive awareness). The small sample size (only 18) could explain the lack of change in some dimensions. However, Psychouli et al. [13] suggested that there was no change in the cultural awareness of students after an international online conferencing collaboration and that the students had a strong desire to participate in more frequent and culturally diverse experiences.

We could further understand the students' feelings, what they learned, and their opinion of this programme by holding focus group interviews to provide some valuable suggestions on future IAH programmes. Three themes were identified, of which the first concerned the learning process. Our study demonstrated that most students recognized that IAH was novel and enjoyable, but they still desired faceto-face cross-cultural communications. A large investigation involving 213,160 undergraduate students from nine universities indicated that undergraduate students who participated in IAH activities reported higher levels of cultural awareness compared with those who had studied abroad [14]. Therefore, they concluded that IAH was just as effective as, if not more effective than, formal study abroad [14]. This might be because when students study abroad, they are in a new environment and normally experience anxiety, which has the potential to negatively influence their communications with others; while when they are in a familiar environment (i.e., their own home, school), they are much more comfortable interacting with people from another culture [15]. Besides, not all students are able to study abroad because of financial issues and limited time. One study found that at most $10 \%$ of students were able to engage in mobility programmes abroad [16].

With regard to the learning outcomes, our study found that students gained new knowledge and increased levels of cultural awareness, which further validated the results of the quantitative data. However, this result came as a surprise to them, which indicated that they might have had few chances to participate in inter-cultural activities or meet people from different cultural backgrounds online or offline before, which was also found in other study [17]. Thus, it is essential to have cross-cultural activities or programmes at school or to integrate IAH in the curriculum, so that international programmes can be available to all students in their home campus [13]. In addition to new knowledge, a few students in this study developed a sense of international social responsibility and cultural sensitivity in clinical practice. 
IAH not only has the potential to prepare students for clinical practice; more importantly, it generates in them a sense of civic responsibility in a global world. COVID-19, as well as other global health events, such as outbreaks of the Ebola virus and SARS, all indicate the need for international communication and collaboration among health workers around the world [8]. Therefore, a sense of international responsibility is needed today more than ever. Our study showed that a four-session IAH programme can potentially develop a sense of international responsibility in undergraduate students, which is similar to the finding of another study [15].

The last theme was about the factors influencing learning outcomes. We found that a micro-movie and active communication were facilitators of the IAH programme. These two factors in fact make the IAH programme a combination of a case-based study and a cross-cultural one, leading to a high level of realism. The student-led approach encouraged the students to positively communicate with and learn from each other. Similarly, a systematic review indicated that a specially designed case study can improve the cultural awareness and competence of nursing students at a low cost [18]. Some students pointed that more focus can be given in the case to the Indonesian culture. This is a valuable suggestion, which will allow us to further improve the IAH programme. Specifically, we need to be alert to placing equal importance on different cultures in future IAH programmes. On the other hand, there were some barriers to the programme, and language was the one mentioned most often, as in other studies $[13,15$, 19]. Language barriers significantly affect communication because people who come from various cultural backgrounds do indeed need more time to process a foreign language to communicate effectively than those from the same cultural background [20]. In addition, language barriers may restrict the depth of interviews if they are conducted in English or another language that is not the mother tongue of the students [15]. As suggested by our students and by the results of another study [21], smaller groups $(n<5)$ may be a solution to the problems posed by language barriers. The time demands of the IAH programme were also not very suitable to the Indonesian students because they were in their final year and might not have had enough time for the programme. This implies that the IAH programme should be tailored to the participants in order to maximize the benefits to be derived from it. In addition, there were some disagreements among the students in our study on the duration of the activities. One study showed that having activities last less than one hour at a time is the most effective duration [13], which needs further study.

To conclude, this study found that a four-session online internationalization at home programme is effective at improving the cultural awareness of students, as validated by qualitative data. Moreover, this programme is enjoyable and has the potential to improve the cultural sensitivity of students in clinical practice and their sense of responsibility in a global world. A micro-movie and active communication are facilitators of the programme, while language barriers, time management, and insufficient prior briefing are barriers, which should be addressed or avoided in future studies.

\section{Strengths And Limitations}


First, this is one of the very few studies to explore students' experience of an IAH programme during a pandemic. It gives a unique understanding of how IAH could work when all face-to-face cultural exchange sessions are suspended. Also, our IAH programme was co-designed by medical and nursing professionals from both of the countries involved. Such a collaboration can make the programme a good fit for the needs of medical and nursing students from both countries. However, this study has its limitations. The participants were all students who had self-enrolled in the course. They were likely to be those who enjoy cultural exchange activities. Our findings may not be fully generalizable to all students in universities in Hong Kong and Indonesia. Also, all of our focus group interviews were conducted by the instructors of the course. This could have affected the equity of the relationship between the interviewer and the participants (rising hierarchy issues). The participants might have felt compelled to provide more desirable feedback in the interview than they would otherwise have given.

\section{Declarations}

\section{Ethics approval and consent to participate:}

The study (involving humans) was performed in accordance with the Declaration of Helsinki. Ethical approval was granted by the Ethics Committee of the Hong Kong Polytechnic University (Reference number: HSEARS20210604001). Informed consent was obtained from all subjects and/or their legal guardian(s) in the study.

\section{Consent for publication:}

Not applicable.

\section{Availability of data and materials:}

The datasets generated and/or analysed during the current study are not publicly available due to the agreement with the subjectsbut are available from the corresponding author on reasonable request.

\section{Competing interests:}

The authors declare they have no competing interests

\section{Funding:}

This project was funded by the Service-Learning and Leadership Office, The Hong Kong Polytechnic University (PolyU).

\section{Authors' contributions}

PK, MMW, IAT, and PPconceptualized the research design and designed the programme. All authors conducted the workshops and assisted in data collection. PK and CY performed statistical analyses and 
qualitative analyses. IAT, $\mathrm{PK}$, and $\mathrm{CY}$ interpreted the data and drafted the manuscript. All authors read and approved the final manuscript.

\section{Acknowledgements}

We would like to express our gratitude to all of the authors for being the instructors of this programme, as well as to all of our research participants for supporting this project.

\section{References}

1. Kripalani S, Bussey-Jones J, Katz MG, Genao I: A prescription for cultural competence in medical education. J GEN INTERN MED 2006, 21(10):1116-1120.

2. Beach MC, Price EG, Gary TL, Robinson KA, Gozu A, Palacio A, Smarth C, Jenckes MW, Feuerstein C, Bass EB et al: Cultural Competence: A Systematic Review of Health Care Provider Educational Interventions. Medical care 2005, 43(4):356-373.

3. Sue S, Zane N, Nagayama Hall GC, Berger LK: The Case for Cultural Competency in Psychotherapeutic Interventions. Annual review of psychology 2009, 60(1):525-548.

4. Khalid J, Ali AJ: Promoting Internationalization at home in ASEAN Higher Education Institutions; A proposed project. 2018.

5. Wit HD: Internationalization of Higher Education: Nine Misconceptions. International Higher Education,(64), 17-19. In.; 2011.

6. Beelen J, Jones E: Redefining internationalization at home. In: The European higher education area. edn.: Springer, Cham; 2015: 59-72.

7. Leung DY, Kumlien C, Bish M, Carlson E, Chan PS, Chan EA: Using internationalization-at-home activities to enhance the cultural awareness of health and social science research students: A mixedmethod study. Nurse Educ Today 2021, 100:104851.

8. Wu A, Leask B, Choi E, Unangst L, de Wit H: Internationalization of Medical Education-a Scoping Review of the Current Status in the United States. Med Sci Educ 2020.

9. Wu A, Kielstein H, Sakurai T, Noel G, Viranta-Kovanen S, Chien C-L, Bernd P: Internationalization of medical education-building a program to prepare future leaders in healthcare. Med Sci Educ 2019, 29(2):535-547.

10. de Wit $\mathrm{H}$, Altbach PG: Internationalization in higher education: global trends and recommendations for its future. Policy Reviews in Higher Education 2021, 5(1):28-46.

11. Borglin G: The value of mixed methods for researching complex interventions. In., edn.; 2015: 29-45.

12. Kumlien C, Bish M, Chan EA, Rew L, Chan PS, Leung D, Carlson E: Psychometric properties of a modified cultural awareness scale for use in higher education within the health and social care fields. BMC medical education 2020, 20(1):406-406.

13. Psychouli P, Collins K, Zadnik M: Internationalization at Home: An Occupational Therapy Synchronous Collaboration Between Cyprus and the United States. Am J Occup Ther 2020, 
74(5):7405205120p7405205121-7405205120p7405205129.

14. Soria KM, Troisi J: Internationalization at home alternatives to study abroad: Implications for students' development of global, international, and intercultural competencies. Journal of studies in international education 2014, 18(3):261-280.

15. Leung DYL, Chan EA, Wong AKC, Reisenhofer S, Stenberg M, Pui Sze C, Lai KH, Cruz E, Carlson E: Advancing pedagogy of undergraduate nursing students' cultural awareness through internationalization webinars: A qualitative study. Nurse Educ Today 2020, 93:104514.

16. Viewpoint - Internationalisation of Medical Education: Hierarchies, Inequalities and Future Directions [https://ipedjournal.com/2015/03/30/]

17. Murden R, Norman A, Ross J, Sturdivant E, Kedia M, Shah S: Occupational therapy students' perceptions of their cultural awareness and competency. Occupational therapy internationa/2008, 15(3):191-203.

18. Qin Y, Chaimongkol N: Simulation With Standardized Patients Designed as Interventions to Develop Nursing Students' Cultural Competence: A Systematic Review. J Transcult Nurs 2021, 32(6):778-789.

19. Chan EA, Lai T, Wong A, Ho S, Chan B, Stenberg M, Carlson E: Nursing Students' Intercultural Learning via Internationalization at Home: A Qualitative Descriptive Study. Nurse Educ Today 2017, 52:34-39.

20. Chen S-J, Hsu C, Caropreso E: Cross-cultural collaborative online learning: When the west meets the east. In: E-Learn: World Conference on E-Learning in Corporate, Government, Healthcare, and Higher Education: 2005: Association for the Advancement of Computing in Education (AACE); 2005: 19311941.

21. Aldrich RM, Johansson KE: U.S. and Swedish Student Learning Through Online Synchronous International Interactions. Am J Occup Ther 2015, 69 Suppl 2:6912350010p69123500116912350015.

\section{Supplementary Files}

This is a list of supplementary files associated with this preprint. Click to download.

- Tables20211228.docx 